\title{
O USO DE TECNOLOGIA EDUCACIONAL E SOCIAL NA FORMAÇÃO DE SANITARISTA
}

\author{
Olga Albuquerque', Maria Hosana Conceição', Maria Fernanda Melis', Felipe Albuquerque ${ }^{2}$, \\ Neusi Berbel ${ }^{3}$ e Cecile Rodrigues ${ }^{4}$ \\ 1 Universidade de Brasília, Brasil.olgamaria@unb.br; hosanac@unb.br; mfernanda.melis@gmail.com \\ ${ }^{2}$ Escola Politécnica da Universidade de São Paulo, Brasil. felipe.moreira.albuquerque@usp.br \\ ${ }^{3}$ Professora aposentada da Universidade Estadual de Londrina - Paraná. neusiberbel@gmail.com \\ ${ }^{4}$ Gestão em Saúde UPE/UAB, Brasil. cecile_rodrigues@hotmail.com
}

\begin{abstract}
Resumo. Introdução: essa pesquisa se situa no contexto de mudanças no pensamento sobre o ensino superior, que apontam a necessidade de formação de profissionais críticos e com capacidade de análise da realidade social. Objetivos: analisar a percepção de estudantes da graduação em Saúde Coletiva da Faculdade de Ceilândia-Universidade de Brasília (SC/FCE-UnB), sobre a utilização de Tecnologia Educacional e Social (TES) relativas ao ensino-aprendizagem na disciplina Atenção Primária à Saúde (APS). Métodos: estudo de caso e análise de conteúdo das respostas ao questionário aberto aplicado aos estudantes de SC. Da leitura do corpus da pesquisa emergiram três grupos de sentidos: TES na formação da identidade profissional; importância da TES na percepção do nexo teoria/prática na APS e importância da TES na interação estudantes/gestores/usuários de Unidades Básicas de Saúde. Resultados: o emprego de TES proporcionou um processo de ação-reflexão-ação, ampliando/acelerando processos complexos de aprendizagem. A experiência pedagógica estimulou a identificação crescente do estudante com sua futura profissão, maior compreensão da relação teoria/prática e percepção da complexidade humana envolvida nos serviços de APS. Conclusões o desafio da articulação da disciplina APS ao mundo real no campo de prática da APS implica a necessidade de boa comunicação entre diferentes instituições/atores sociais para alcançarem objetivos pedagógicos comuns.
\end{abstract}

Palavras-chave: Tecnologia Educacional; Tecnologia Culturalmente Apropriada; Formação Profissional em Saúde; Atenção Primária à Saúde.

\section{EDUCATIONAL AND SOCIAL TECHNOLOGY AS A MEANS OF BRIDGING THE GAP BETWEEN THEORY AND PRACTICE}

Abstract. Introduction: this research is situated in a context of changes regarding thinking about higher education, which point towards the necessity of educating critical professionals. Aim: to analyze the Public Health students' perception at the Public Health at the University of Brasilia, in utilizing social/educational technology (SET) related to the teaching-learning process of the Primary Health Care (PHC) class. Methods: case study and content analyses of responses to questionnaire of the PHC discipline applied to the students. The research's answers were threefold: SET played a part in molding professional identity; importance of SET in the perception of the nexus theory/practice in PHC and the importance of SET in the interaction between students, managers and users of Basic Health Units. Results: the use of SET allowed for an action-reflection-action process, amplifying/accelerating complex teaching processes. The pedagogical experience stimulated a growth in the students' identification with their future profession, a better understanding of the relationship between theory/practice and the perception of human complexity involved in PHC. Conclusions the challenge of articulating the PHC discipline with the real world in a practical manner, on the field of PHC, entails the need of proper communication between different social institutions/actors in order to achieve pedagogical common goals.

Keywords: Educational Technology; Culturally Appropriate Technology; Health Human Resource Training; Primary Health Care. 


\section{INTRODUÇÃO}

A habilidade de tomar decisões sobre o quê e como ensinar, considerando quem, por quê, quando e onde se ensina, caracteriza o conceito de didática. Sua utilização não visa apenas métodos e estratégias de aprendizado, nem uma simples adesão a novas tecnologias ou aumento de produtividade no trabalho. Mais do que isso é importante que o ensino se coloque a serviço do desenvolvimento pessoal e crítico do(a) estudante e da sociedade (Sant'Anna \& Menegolla, 1989).

As deficiências na formação do professor no contexto do ensino superior têm sido reconhecidas. A crença de que para ser um bom professor bastariam sólidos conhecimentos na disciplina que leciona e boa comunicação, não é mais aceitável. Estudos pedagógicos realizados com estudantes universitários revelam que uma das maiores críticas ao ensino tem sido a "falta de didática dos professores" (Gil, 2012).

Desse modo, torna-se relevante refletir sobre estratégias didáticas, especialmente no cenário da formação em saúde pública, considerando que o Sanitarista constitui um novo ofício em saúde em fase de consolidação de sua identidade profissional (Silva \& Pinto, 2013; Albuquerque et al., 2018; Silva, Pinto \& Teixeira, 2018). Segundo Katajavouri, Lindblom-Ylänne \& Hirovonen (2006) o reforço dessa identidade profissional resulta, também, da aplicação dos conhecimentos teóricos adquiridos e aplicados no mundo real em situações de trabalho.

Para McCrone, O’Beirne, Sims \& Taylor (2015, tradução nossa) uma das principais características do ensino profissional é o modo como o ensino e a aprendizagem se relacionam com o local de trabalho ou como são contextualizados no exercício da profissão. O cenário de prática define a natureza da aprendizagem, abrangendo a contextualização do conteúdo do curso e as abordagens pedagógicas. Dessa forma, inclui-se o local de trabalho e garante-se que o estudante compreenda o que é estudado.

Diferentes estudos (Guile \& Evans, 2010; Vendruscolo, Prado \& Kleba, 2016) abordam a importância da conexão entre teoria/prática em ambiente institucional com vistas à "adequação do processo de formação às necessidades do Sistema Único de Saúde". Considerando que o conhecimento prático é adquirido mediante a participação dos estudantes em um ambiente de trabalho no qual a teoria possa ser aplicada, e mesmo questionada, a parceria pedagógica entre a universidade e serviços que oferecem Atenção 
Primária à Saúde (APS) tem sido fundamental. O processo de aprendizagem nesse cenário pode facilitar uma interação potencialmente construtiva entre a teoria e a prática. Tal parceria é o fundamento sobre o qual a teoria debatida em sala passa a fazer sentido e cria um cenário favorável ao ensino-aprendizagem.

Essa dinâmica de aprendizagem se insere no conceito de tecnologia educacional aqui entendida como "o estudo e a prática ética de facilitar a aprendizagem e melhorar o desempenho (performance) por meio da criação, do uso e do gerenciamento apropriado de processos tecnológicos e de recursos". Nos anos sessenta a concepção de tecnologia educacional estava focada em "controlar o processo de aprendizado". Atualmente a ênfase é colocada em "facilitar a aprendizagem" fornecendo "o espaço do problema e as ferramentas cognitivas para explorá-lo [...] em ambientes imersivos para apoiar o aprendizado" (Januszewski \& Molenda, 2008, p. 04).

No Distrito Federal (DF) a Estratégia Saúde da Família (ESF) constitui a estratégia de orientação do modelo de Atenção Primária à Saúde (APS) instituída pela Política de Atenção Primária à Saúde do Distrito Federal/Brasília (Distrito Federal, 2017). Sua implementação se baseia nas seguintes diretrizes: o acolhimento, a classificação de risco, a resolutividade, a territorialização, a adscrição de clientela, a garantia de acesso e a ordenação da rede de saúde.

A adscrição da clientela constante da PAPS-DF (Distrito Federal, 2017, p. 03) preconiza que "cada equipe deve promover o cadastramento e o acompanhamento da população sob sua responsabilidade, por meio de ações na unidade de saúde, na comunidade ou em visitas domiciliares, utilizando as informações para o planejamento de seu trabalho e para o desencadeamento de ações de outros níveis da gestão".

Esta pesquisa se situa no contexto de mudanças no pensamento sobre a formação no ensino superior, que apontam para a necessidade de formação de profissionais críticos com capacidade de análise da realidade social. Assim, é importante que caminhos metodológicos percorridos com essa intenção pelos processos de ensino-aprendizagem sejam analisados.

Considerando que a graduação em Saúde Coletiva é um curso em fase de consolidação, a pergunta a ser respondida pelo estudo é: quais características de TES foram reconhecidas pelos estudantes como as mais adequadas ao ensino-aprendizagem na disciplina Atenção Primária à Saúde? 
Objetivo: analisar a percepção de estudantes do curso de graduação em Saúde Coletiva, sobre a adoção de Tecnologia Educacional e Social (TES) no ensino-aprendizagem na disciplina de Atenção Primária à Saúde (APS).

\section{METODOLOGIA}

Adotou-se a pesquisa qualitativa do tipo estudo de caso cuja principal característica é ser "uma investigação empírica que pesquisa um fenômeno contemporâneo dentro de seu contexto da vida real, especialmente quando os limites entre o fenômeno e o contexto não estão claramente definidos; enfrenta uma situação tecnicamente única em que haverá muito mais variáveis de interesse do que pontos de dados e, como resultado, baseia-se em várias fontes de evidência (Yin, 2005, p. 13) [...] e beneficia-se do desenvolvimento prévio de proposições teóricas para conduzir a coleta e análise dos dados. É uma estratégia de investigação mais adequada quando queremos saber o 'como' e o 'porquê' de acontecimentos atuais sobre os quais o investigador tem pouco ou nenhum controle (Yin, 2005, p. 9).

Segundo Stake (2000, p. 445, tradução nossa) o estudo de caso é considerado intrínseco porque "ilustra um caso particular e possibilita o entendimento mais profundo de um caso específico". E, no caso deste estudo, é heurístico porque acrescenta compreensão à prática pedagógica na disciplina APS, ampliando a experiência da professora ou confirmando aspectos já conhecidos de sua prática. Nessa pesquisa se delineou um conjunto de ações intencionais de ensino-aprendizagem promovidas em sala de aula e no cenário de prática institucional, que se desenvolveram nos semestres 2018/2, 2019/1 e 2019/2 com sessenta e sete estudantes.

Ministrada no quarto semestre do curso de graduação em Saúde Coletiva (SC) na Faculdade de Ceilândia/Universidade de Brasília (FCE/UnB), a disciplina APS de 40 horas vem sendo ofertada, ininterruptamente, desde 2014 até o presente. Seu desenvolvimento busca materializar a articulação "entre teoria e prática", a interlocução com egressos exercendo função de sanitaristas nos serviços de saúde, bem como a "indissociabilidade entre ensino, pesquisa e extensão". Essa disciplina está integrada ao Projeto de Extensão de Ação Contínua "Tecnologia Educacional e Social como dinamizadoras na formação em saúde". Tudo isso está previsto no Projeto Político Pedagógico do Curso de Saúde Coletiva (Universidade de Brasília [UnB], 2019). 
A conversão do modelo tradicional de APS do DF em ESF está consubstanciada na Portaria 77 (Distrito Federal, 2017). Adicionalmente, a Portaria 78 (Distrito Federal, 2017) facultava aos profissionais de saúde sua realocação na APS ou em outros pontos da atenção à saúde. Isso permitiu "a composição de 442 equipes de Saúde da Família (eSF) e a ampliação da cobertura populacional para $69,1 \%$ ".

Em seu empenho para aumentar a população assistida, a Secretaria Estadual de Saúde DF admite que a ausência dos Agentes Comunitários de Saúde (ACS) nas eSF corresponde ao seu "principal desafio para promover o cadastramento de todas elas" (Corrêa et al., 2019, p. 2037). A parceria da disciplina APS com o serviço de saúde do DF se situa nessa condição de escassez de ACS para efetuar o registro dos residentes nos territórios adstritos às Unidades Básicas de Saúde (UBS).

Desse modo os estudantes da disciplina APS visitam as residências da microárea, interagem com seus moradores e preenchem os cadastros para registrar as pessoas que constituem a população adscrita à UBS 16. Nessa atividade eles são acompanhados de perto pelas ACS. Uma regra básica de comportamento para atuar nesse cenário é o respeito devido a cada uma dessas pessoas: as ACS, os usuários com quem entram em contato e os outros servidores da UBS. Na sequência desse processo de trabalho, os estudantes procedem à inserção dos dados obtidos no Sistema de Informação da Atenção Básica (SISAB), supervisionados pelos profissionais do setor de Vigilância em Saúde.

Essa pesquisa utilizou TES específicas como ferramenta pedagógica para uma formação profissional contextualizada e inserida nas diferentes atividades na APS em Ceilândia. A efetivação dessas TES consistem em: leituras e sínteses dos textos sobre APS; apresentação da síntese destes artigos científicos que adotaram práticas inter-relacionadas à APS com base em critérios de avaliação pré-definidos e em sua vivência na APS; apropriação da legislação vigente no confronto com sua materialização no cenário de práticas; reflexão efetuada a partir das vivências no campo da APS; interação com os moradores da microárea e com servidores da UBS; e, interlocução dos estudantes com gestores e egressos que já atuam como Sanitaristas. As perguntas elaboradas por eles ao apresentarem as sínteses em sala de aula, guiam o diálogo nas rodas de conversa.

Em cada semestre a sala de aula foi o local onde se realizaram as cinco rodas de conversa com a participação de gestores e egressos de SC, com duração de duas horas e a participação de vinte e dois estudantes em média, dos quais $76 \%$ eram do gênero feminino. 
A professora moderou essas conversas coletivas que partiam das questões elaboradas pelos estudantes apoiados nas leituras e em suas vivências nas UBS.

O registro dos dados se originou das frases dos estudantes ao avaliarem a disciplina APS, sequencialmente, ao fim dos três semestres e compuseram o corpus sobre o qual efetuouse a análise de conteúdo. Uma das perguntas propostas aos estudantes para sua avaliação da TES adotada no contexto da APS contemplou "a contribuição da disciplina APS ao seu processo de formação profissional" no campo da saúde pública. A cada vez que se repetia a aplicação destas perguntas a professora retirava-se da sala para que os estudantes pudessem expressar-se sem qualquer tipo de constrangimento.

A análise de conteúdo se serviu do tema como regra de recorte como a "unidade de significação que se liberta naturalmente de um texto analisado segundo certos critérios relativos à teoria que serve de guia à leitura". O emprego do tema como unidade de registro se ajusta à investigação de "motivações de opiniões, de atitudes, de valores, de crenças, de tendências, etc." (Bardin, 2011, p. 135). Isso parece adequado ao propósito do estudo, que é construir conjuntamente com os estudantes, ao longo dos semestres o aprimoramento da disciplina APS.

A organização dos dados do estudo iniciou com a transcrição das frases, que constituíram as unidades de registro. Em seguida fez-se a leitura flutuante para formulação de hipóteses provisórias quanto às categorias levantadas inicialmente. Em seguida, a pesquisadora ordenou os dados a partir de uma leitura exaustiva e repetida com vistas à codificação e identificação de núcleos de sentido. Na análise final fez-se a triangulação com o referencial teórico e o material coletado. Os resultados apresentam as unidades de registro dos estudantes identificados pela letra "E" seguida do número associado a cada um dos acadêmicos que efetuou as avaliações e passou a compor o corpus da pesquisa.

\section{RESULTADO}

É importante destacar que no DF a implantação do modelo de APS operacionalizou a conversão do modelo tradicional de APS em ESF com prioridade para as áreas de maior vulnerabilidade social (Corrêa et al., 2019). Isso teve início em 2016 e, no ano seguinte, a publicação da Portaria 78 (Distrito Federal, 2017) propunha aos profissionais contratados a adesão ao novo modelo de atenção. A estes profissionais foi facultada a opção de serem "relotados em unidades de saúde de outros níveis de atenção, não necessariamente na 
mesma Região de Saúde, em função da necessidade da população e respeitadas as atribuições de seu cargo."

Assim, desde o início do semestre letivo, os estudantes visitavam as UBS para observar traços da realidade existente e confrontar as facetas desta realidade com aspectos teóricos que estruturam a implementação da PAPS-DF (Distrito Federal, 2017). Da leitura do corpus desta pesquisa emergiram sentidos relativos às estratégias de TES adotadas no ensinoaprendizagem da APS categorizados em 3 grupos: a) TES no processo formação da identidade profissional; b) importância da TES para a percepção do nexo entre teoria/prática na APS; c) importância da TES na interação entre estudantes, gestores e usuários de UBS.

\subsection{Tecnologia educacional e social no processo de formação da identidade profissional}

O repertório de frases destacadas a seguir apontam a relevância da TES para solidificar a autopercepção como profissional "mais capacitada para lidar com problemas" (E1), "sensata e realista" (E2), "atento às necessidades da população" (E3), "capaz de enfrentar dificuldades" (E4), [preparado para] "exercer meu papel como gestor" (E5), (E6).

A percepção geral dos estudantes sobre o uso das estratégias de TES utilizadas pela disciplina de APS foi expressa das seguintes formas: "a APS abriu meus olhos ao meu futuro campo de atuação [...] as dificuldades que poderei enfrentar e como resolvê-las" (E6). Outro estudante manifestou que a APS contribuiu para uma "visão mais ampliada do Sistema de Saúde, especificamente a Atenção Básica, com um olhar de profissional' (E7).

“ $A$ [disciplina] APS foi de 'super' contribuição para minha formação em Saúde Coletiva, os conhecimentos das leis e portarias trouxeram um conhecimento mais profundo para uma gestão qualificada e de competência nas Unidades de Saúde" (E8). Outra expressão relevante: "A APS trouxe para o meu processo de formação pessoal uma paixão pela área, uma auto-descoberta como área de atuação, me fez enxergar como nós profissionais de Saúde Coletiva somos fundamentais na ponta, na prestação de serviços" (E9). É possível observar que, em alguns momentos, os estudantes se referem à disciplina APS e, em outros, eles se reportam à APS. A PAPS-DF (Distrito Federal, 2017) determina que este ponto da atenção à saúde seja a "porta de entrada" e o "contato preferencial dos usuários com o sistema de saúde". 
Apesar da dificuldade representada pela leitura inabitual da legislação, os estudantes admitem: "pude aprender melhor sobre a APS e como aplicar esses conhecimentos na UBS, na gestão, intervir segundo as Portarias" (E10). Outro estudante se percebeu exercendo sua profissão na APS: "foi incrível, eu finalmente entendi as possibilidades de meu futuro como gestora, a apropriação do conhecimento (...) essa disciplina me inspira a ser a melhor sanitarista possível' (E12).

Ao partir do conhecimento de um campo concreto de trabalho do Sanitarista os estudantes, de maneira geral, desenvolveram uma identificação crescente com a futura profissão. Ao longo dos semestres as estratégias de TES adotadas na disciplina APS suscitam discussões em sala de aula acerca dos principais desafios a serem enfrentados pelos estudantes em sua futura profissão. As avaliações realizadas por eles, descritas acima, repercutem a dinâmica adotada na disciplina APS.

Os fragmentos expressivos dos estudantes de SC corroboram a pesquisa de Sisson (2009). Nela os estudantes apontam a identidade profissional e a aquisição de valores sociais como resultado da prática vivenciada e realizada: "aprendi a me colocar no lugar do tomador de decisão e analisar a situação como um profissional" (E13). Para outros as visitas às UBS despertaram o sentimento de identificação com a decisão apoiada na "legislação para a tomada de decisão" (E12); "me fez pensar como gestor" (E14), "como profissional de saúde na APS, como tomador de decisão" (E15).

E ainda, "A APS trouxe uma perspectiva de conhecimento na função de fazer, delegar e de gestor de saúde" (E16). Tais características, essenciais nas atividades realizadas, desvelam ganhos importantes na formação dos acadêmicos na disciplina. No estudo de Katajavouri, Lindblom-Ylänne \& Hirovonen (2006), os estudantes afirmaram que o período de prática os preparava para a vida profissional e Ihes permitia ir trabalhar depois da formatura.

As atividades em sala de aula representaram um duplo desafio: impulso para os estudantes se apropriarem dos principais conceitos que dão sustentação à APS e a compreensão da lógica da legislação estruturante do modelo da APS, PAPS-DF (Distrito Federal, 2017) e Política Nacional de Atenção Básica (Brasil, 2017), como norteadora das decisões. A disciplina Atenção Primária à Saúde foi incorporada com este nome às trilhas de aprendizagem do curso de Saúde Coletiva (SC). Ao longo desse texto observa-se que alguns estudantes empregam o termo Atenção Básica (AB). Isso acontece porque ambos os 
normativos, a PAPS (Distrito Federal, 2017) e a PNAB (Brasil, 2017) admitem os dois termos, AB e APS, para designar este ponto da atenção em saúde.

"Como profissional devo sempre ser pautada pelos regulamentos [...] ter esperteza e feeling na tomada de decisão, além de conhecer muito bem o meu objeto de trabalho: teoria + senso crítico + vínculo" (E11). A aquisição dos saberes profissionais mesclou a teoria, a legislação, as práticas reflexivas e a sinergia com o trabalho propiciado pelas vivências nesse trajeto formativo.

O conjunto de enunciados dos estudantes desvelam que "é no encontro entre o mundo da formação e o mundo do trabalho que o aprender e o ensinar se incorporam ao cotidiano" (Ramos, Barros \& Ferraço, 2004). O Projeto Político Pedagógico [PPP] (Brasil, 2019) do curso de SC compreende quatro eixos: o modo de vida, os sistemas biológicos, as especificidades do fazer em SC e os cenários e práticas em SC. O eixo que trata das "especificidades do fazer em SC enfatiza aqueles aspectos, conteúdos e abordagens, voltados à qualificação da ação dos estudantes nas questões diretamente implicadas na prática de estruturação, organização e gestão da atenção em saúde e na consolidação do Sistema Único de Saúde." Os ângulos de visão trazidos pelos estudantes apontam para uma convergência aos preceitos contidos no PPP (Brasil, 2019).

\subsection{Importância da TES para a percepção do nexo entre teoria/prática na APS}

A atuação dos estudantes no campo, o cadastramento na ESF e o subsequente registro dos dados no SISAB gerou uma disposição favorável à APS, que se constitui na primeira interface dos usuários com o sistema de saúde. Isso parece ter sido importante para a interligação entre teoria e prática nos cenários de aprendizagem propostos.

Referindo-se a essas atuações, dentre as avaliações efetuadas pelos estudantes, encontram-se enunciados como "conhecimento e vivência da realidade da APS [...] vivência na UBS como prática fundamental" (E17), a "aplicação da teoria na prática" (E18), o "vínculo entre teoria e prática" (E19) e o entendimento "como funciona na teoria e na prática a APS" (E15), além de muitas outras expressões enfatizando o nexo identificado entre teoria e prática.

Nesse sentido os estudantes manifestaram sua percepção sobre o tópico do PPP (Brasil, 2019) referente aos "cenários e práticas em Saúde Coletiva" através das seguintes frases: 
"A [disciplina] APS me deu uma visão prática do que acontece na vida real" (E20). Tal perceção está em conformidade com a pesquisa de Lave (1993). Nela o autor salienta que "o conhecimento prático é adquirido trabalhando e participando de um ambiente de trabalho onde a teoria possa ser aplicada. E, por consequência, o conhecimento prático é socialmente situado e socialmente produzido".

"A [disciplina] APS aprofundou meus conhecimentos de APS e também nos colocou como gestores ao nos dar leis e documentos que regem nossa atuação e fazendo a conexão da teoria com a prática" (E11). Essa compreensão desvelada na frase desse estudante advém do discernimento quanto à importância do normativo legal como base para a tomada de decisão do gestor.

"Por fazer a ligação da teoria com a prática [a disciplina APS] me fez enxergar necessidades e ter um olhar mais amplo em relação à sociedade" (E9). "Trouxe coisas importantes como a experiência de vivenciar a teoria com a prática e como nossa formação é importante para a APS. Uma das poucas disciplinas que faz os alunos vivenciarem teoria e prática" (E21). Na proposta pedagógica da disciplina APS os saberes são estimulados/mobilizados mediante a elaboração de sínteses dos artigos interconectados à APS. Ao apresentarem essas sínteses com base nos critérios de avaliação estabelecidos previamente, eles fazem a inter-relação com aspectos das Portarias PAPS-DF (Distrito Federal, 2017) e PNAB (Brasil, 2017). Sua exposição subsidia a discussão e evidencia a importância da legislação para a tomada de decisão do gestor.

No estudo de Katajavouri, Lindblom-Ylänne \& Hirovonen (2006), os resultados mostraram que, durante o período de prática, muitos estudantes sentiram que eram capazes de vincular seu conhecimento teórico à sua atividade no local de trabalho e, assim, obter uma imagem coerente da interconexão entre os aspectos práticos e teóricos. Depois de se familiarizarem com os aspectos práticos da profissão, o conhecimento teórico tomou forma concreta, e os estudantes relataram que agora perceberam como as questões técnicas estavam interligadas entre si.

\subsection{Importância da TES na interação entre estudantes, gestores e usuários de UBS}

Inúmeras palavras remetem à interação e à sinergia dos estudantes em sua relação com os sujeitos no campo de trabalho: "diálogo" (E4), "importância do trabalho em equipe" (E6, E19, 
E20, E22, E23), "vínculo" (E11), "responsabilidade" (E17, E18, E19, E23 ), "empatia" (E15, E17, E18, E20, E21, E24, E25, E26, E29, E30), “compreensão" (E26), “respeito" (E28), "esperança, compaixão" (E30).

A vivência no campo de práticas proporcionada pela atuação no cenário real de uma UBS parece ter produzido importantes reflexões nos estudantes sobre questões relativas à complexidade humana envolvida neste local de trabalho, como indicam as frases abaixo: "com as saídas de campo pude ter uma melhor relação com os demais sujeitos da sociedade, saber ouvi-los e ajudá-los no que for necessário" (E31) e "a disciplina [APS] trouxe uma evolução pois fui desenvolvendo a capacidade de construir relacionamentos interpessoais e aprendendo a lidar com as pessoas" (E32).

"A [disciplina] APS me trouxe a capacidade de compreender os profissionais de saúde, como 'funciona' seus processos de tomada de decisão para lidar com as dificuldades e demandas da população" (E15). E ainda [ajudou a] "me tornar uma melhor profissional, com mais habilidade de escuta, sendo mais humanizada e acolhedora, com melhor compreensão dos processos sociais e do ambiente no qual estarei inserida" (E33).

"A ida à UBS me fez ter contato com uma realidade que eu ainda não conhecia. Me ajudou com valores pessoais como respeito, empatia, solidariedade e 'prestatividade'. Valores que ajudam muito nas relações sociais" (E34). Nesse contexto, outra forma de aproximação da disciplina APS com os serviços de APS ocorre semestralmente, através de rodas de conversa com gestores de APS. Elas constituem mais uma forma de promover o avizinhamento dos estudantes ao cotidiano da profissão de Sanitarista e se configuram na interlocução com gestores e com egressos do curso de Saúde Coletiva exercendo atividade na APS.

"As rodas de conversa com os gestores acolhendo as experiências que trouxeram, agregou conhecimento" (E35). Muito apreciadas pelos estudantes, as Rodas de Conversa "consistem em um método de participação coletiva de debate acerca de determinada temática em que é possível dialogar com os sujeitos, que se expressam e escutam seus pares e a si mesmos por meio do exercício reflexivo" (Moura \& Lima, 2014). Nesses momentos, a participação intensa e interessada manifesta sua empatia com os egressos de SC já trabalhando nos diferentes pontos de atenção à saúde. Desvelam também a relevância dos assuntos tratados que os envolvem diretamente em seu futuro como Sanitaristas, como enfatizou 
(E36): "em especial a presença dos egressos de Saúde Coletiva e dos gestores trouxeram uma perspectiva de futuro".

O conhecimento da percepção dos estudantes sobre a aprendizagem na disciplina de APS constitui um recurso docente para reflexão acerca do desenvolvimento semestral da disciplina, com o objetivo de avaliar criticamente a prática pedagógica e, consequentemente, para seu aprimoramento progressivo. Esse tipo de contribuição gera uma sinergia de esforços e de aprendizagem recíproca. Gera ao mesmo tempo a reflexão crítica, matriz do exame contínuo das "experiências de ensino como base para a avaliação, a tomada de decisão e como fonte de mudança" (Bartlett, 1990, p. 4).

\section{CONCLUSÕES}

Na percepção dos estudantes o emprego de Tecnologia Educacional e Social proporcionou um processo de ação-reflexão-ação, ampliando e acelerando processos complexos de aprendizagem. A experiência pedagógica estimulou uma identificação crescente do estudante com a futura profissão, uma maior compreensão da interconexão teoria e prática, bem como a percepção da complexidade humana envolvida nos serviços de Atenção Primária à Saúde.

A articulação da disciplina APS ao mundo real de trabalho no âmbito da Atenção Primária à Saúde tem sido desafiadora e implica a necessidade de boa comunicação entre diferentes instituições e atores sociais para alcançarem objetivos pedagógicos comuns. A opção pedagógica adotada retrata a materialização de um novo currículo como está previsto no Projeto Político Pedagógico do curso de Saúde Coletiva. E, acima de tudo, representa um novo pensamento na formação de profissionais de saúde.

A adoção da metodologia de Estudo de Caso nessa pesquisa propiciou o aprofundamento do contexto de uma disciplina ministrada na educação superior pública. Sua principal contribuição foi ter enfocado a TES aplicada ao campo de formação de um novo profissional de saúde, o Sanitarista, que se encontra em fase de consolidação. Seu desenvolvimento impacta o exercício da docência, o estabelecimento de parcerias com os serviços de saúde e o exercício profissional na APS. Dentre as estratégias adotadas na disciplina APS, a avaliação efetuada ao final dos semestres proporciona aprendizagem docente em seu relacionamento com os estudantes e com outros atores sociais envolvidos, bem como na reelaboração do plano de ensino. 
É possível afirmar que, com a TES adotada na disciplina, vai ocorrendo uma aproximação gradativa de ações características de Metodologias Ativas, tão indicadas para a área da saúde. Dentre estas ações encontram-se: a articulação direta dos estudantes com o futuro campo de trabalho; a observação direta de problemas/dificuldades que ali ocorrem e que servirão como base de reflexão pessoal e coletiva; a interligação entre a teoria estudada e a prática observada. De um lado verifica-se o engajamento do aluno em relação a novas aprendizagens, pela compreensão e pelo interesse. De outro cria-se a condição para ampliar suas possibilidades de exercitar a liberdade e a autonomia na tomada de decisões, preparando-se para o exercício profissional futuro.

Tudo isso acarreta a reflexão e o aprimoramento da disciplina APS que vão sendo construído ao longo do tempo. Os resultados deste estudo contribuem para o aprimoramento de práticas pedagógicas inovadoras no ensino superior.

\section{REFERÊNCIAS}

Albuquerque et al. (2018). Uso de tecnologia Social na constituição do conselho gestor de unidade de saúde. RISTI - Revista Ibérica de Sistemas e Tecnologias de Informação, (28), 41-56.

Bardin, L. (2011). Análise de conteúdo (3. reimp.). Lisboa: Edições, 70.

Bartlett, L. (1990). Teacher development through reflective teaching. In J. C. Richards \& D. Nunan (Eds.). Second language teacher education (p. 202-214). New York: Cambridge University Press.

Brasil (2017). Portaria de Consolidação $n^{\circ}$ 02, de 28 de setembro de 2017. Aprova a Política Nacional de Atenção Básica. Recuperado em 24 abril, 2020 , em http://bvsms.saude.gov.br/bvs/saudelegis/gm/2017/prc0002_03_10_2017.html

Brasil (2019). Projeto Político-Pedagógico. Recuperado em 24 abril, 2020, em http://fce.unb.br/images/documentos/graduacao/saudecoletiva/ppp/ppc_saude_coletiva.pdf.

Corrêa D. S. R. et al. (2019). Movimentos de reforma do sistema de saúde do Distrito Federal: a conversão do modelo assistencial da Atenção Primária à Saúde. Ciência \& Saúde Coletiva, 24(6), 2031-2041.

Distrito Federal (2017). Protocolo de acesso de atenção primária à saúde do Distrito Federal - PAPS/DF. Recuperado em 24 abril, 2020, em http://www.saude.df.gov.br/wp-conteudo/uploads/2018/04/ProtocoloVers\%C3\%A3o-final-aprovada-na-CPPAS-10.04-apresentada-ao-gab.pdf.

Gil, A. C. (2012). Didática do ensino superior (1. ed., 7 reimpr.). São Paulo: Atlas.

Guile, D. \& Evans, K. (2010, October). Putting knowledge to work: re-contextualising knowledge through the design and implementation of work-based learning at higher education levels. Recovered in 24 April,2020, on https://s3.eu-west-2.amazonaws.com/assets.creode.advancehe-documentmanager/documents/hea/private/putting-knowledge-to-work_1568037385.pdf.

Januszewski, A. \& Molenda, M. (2008). Definition. In A. Januszewski \& M. Molenda (Eds). Educational technology: a definition with commentary (1st. ed., p. 195-211). New York: Lawrence Earlbaum Associates.

Katajavuori, N., Lindblom-Ylänne, S. \& Hirvonen, J. (2006). The significance of practical training in linking theoretical studies with practice. Higher Education, (51), 439--464. 
Lave, J. (1993). The practice of learning. In S. Chaiklin \& J. Lave (Eds.). Understanding practice: perspectives on activity and context (p. 3-32). Cambridge: Cambridge University Press.

McCrone, T., O'Beirne, C., Sims, D. \& Taylor, A. (2015, November). A review of technical education. Recovered in 24 abril, 2020, on https://www.nfer.ac.uk/media/1584/aoct01.pdf.

Portaria $n^{\circ}$ 77, de 14 de fevereiro de 2017 (2017). Estabelece a Política de Atenção Primária à Saúde do Distrito Federal. Diário Oficial do Distrito Federal. Brasília, DF: Secretaria de Estado de Saúde.

Portaria no 78, de 14 de fevereiro de 2017 (2017). Regulamenta o art. 51 da Portaria $n^{\circ}$ 77, de 2017, para disciplinar o processo de conversão da Atenção Primária à Saúde do Distrito Federal ao modelo a Estratégia Saúde da Família. Diário Oficial do Distrito Federal. Brasília, DF: Secretaria de Estado de Saúde.

Ramos, M. C., Barros, M. E. B. \& Ferraço, C. E. (2016). As redes cotidianas dos currículos na formação dos trabalhadores da saúde. In: F. Sodré, M. A. C. Andrade, R. C. D. Lima \& A. C. P. Garcia. Formação em Saúde: práticas e perspectivas no campo da saúde coletiva (p. 30-56). Vitória: EDUFES.

Sant'Anna, I. M. \& Menegolla, M. (1989). Didática: aprender a ensinar [Coleção Escola e Participação]. São Paulo: Loyola

Silva, O. S. \& Pinto, I. C M. (2013). Construção da identidade dos atores da saúde coletiva no Brasil: uma revisão da literatura. Interface - Comunicação, Saúde, Educação, 17(46), 549-560.

Silva, O. S., Pinto, I. C M. \& Teixeira, C. F. (2018). Identidade profissional e movimentos de emprego de egressos dos cursos de graduação em Saúde Coletiva . Saúde em Debate, 42(11), 799-808.

Sisson, M. C. (2009). Implantação de programas e redefinição de práticas profissionais. Revista Brasileira de Educação Médica, 33 (Suppl. 1), 92-103.

Stake R. (2000). Case studies. In. Denzin NK e Lincoln YS. Handbook of qualitative research (2nd ed.). California: Sage.

Vendruscolo, C., Prado, M.C. \& Kleba, M. E. (2016). Integração ensino-serviço no âmbito do Programa Nacional de Reorientação da Formação Profissional em Saúde. Ciência \& Saúde Coletiva, 21(9), 2949-2960.

Yin, R. K. (2005). Estudo de caso: planejamento e métodos. Porto Alegre: Bookman. 